\title{
BMJ Open Effectiveness of female condom in preventing HIV and sexually transmitted infections: a systematic review protocol
}

\author{
Ruth KB Mome, ${ }^{1}$ Alison B Wiyeh, ${ }^{2}$ Eugene J Kongnyuy, ${ }^{3}$ \\ Charles Shey Wiysonge $e^{2,4,5}$
}

To cite: Mome RKB, Wiyeh $A B$, Kongnyuy EJ, et al. Effectiveness of female condom in preventing HIV and sexually transmitted infections: a systematic review protocol. BMJ Open 2018;8:e023055. doi:10.1136/ bmjopen-2018-023055

- Prepublication history for this paper is available online. To view these files, please visit the journal online (http://dx.doi. org/10.1136/bmjopen-2018023055).

RKBM and ABW contributed equally.

Received 19 March 2018 Revised 29 May 2018 Accepted 29 June 2018

Check for updates

(C) Author(s) (or their employer(s)) 2018. Re-use permitted under CC BY-NC. No commercial re-use. See rights and permissions. Published by BMJ.

For numbered affiliations see end of article.

Correspondence to

Dr Alison B Wiyeh;

wberiliy@yahoo.co.uk

\section{ABSTRACT}

Introduction The HIV pandemic continues to evolve with young women being the most vulnerable group to acquire infection. The presence of sexually transmitted infections (STIs) further enhances HIV susceptibility and also leads to long-term complications such as infertility and cervical cancer. The female condom is a self-initiated method for STI and HIV prevention but there are controversies on its effects. We aim to assess the effectiveness, safety and acceptability of the use of female condoms for prevention of STI and HIV acquisition among women.

Methods and analysis We will search Cochrane Central Register of Controlled Trials, PubMed, EMBASE, Scopus, WHO International Clinical Trials Registry and reference lists of relevant publications for potentially eligible studies. We will screen search outputs, select eligible studies, extract data and assess risk of bias in duplicate; resolving discrepancies through discussion and consensus or arbitration. We will combine data from clinically homogenous studies in a fixed effect meta-analysis and assess the certainty of the evidence using the method for Grading of Recommendations Assessment, Development and Evaluation. We registered the planned systematic review with the International Prospective Register of Systematic Reviews (PROSPER0) in March 2018 and will finalise the search strategy in August 2018; conduct the searches and select eligible studies between August and October 2018; and collect data, conduct statistical analyses and prepare and submit the manuscript for consideration by a peer-reviewed journal between November 2018 and April 2019.

Ethics and dissemination We will use publicly available data; hence no formal ethical approval is required for this review. We will disseminate the findings of this review through conference presentations and publication in an open-access peer-reviewed journal.

PROSPERO registration number CRD42018090710.

\section{INTRODUCTION}

The disease burden resulting from unsafe sex, including HIV infection and other sexually transmitted infections (STIs), has profoundly impacted low-income and middle-income regions, especially Sub-Saharan Africa. ${ }^{1}$ The
Strengths and limitations of this study

- We will conduct a comprehensive search for potentially eligible, completed and ongoing trials which would ensure that we obtain an unbiased summary of intervention effects.

- This review will include non-randomised trials which are more prone to bias than randomised trials. However, to minimise the effect of the bias, we will perform subgroup analyses by study design.

- The Grading of Recommendations Assessment, Development and Evaluation approach will be used to assess the certainty of the evidence.

HIV pandemic continues to evolve in both magnitude and diversity, with over 40 million infections worldwide, with young women aged 15-24 being 2.5 times more likely to be infected than young men. ${ }^{2}$ In many cases, STIs go undiagnosed and eventually lead to longterm complications such as infertility and cervical cancer. In addition, the presence of an STI enhances HIV susceptibility. ${ }^{3-5}$ Several interventions exist for the prevention of HIV and STIs such as the male condom. Although male condoms are effective in reducing HIV and STIs transmission, ${ }^{6}$ the subordinate status of women in many countries, especially in Sub-Saharan Africa, makes negotiating male condom use with partners especially difficult. ${ }^{78}$ Hence, women remain particularly vulnerable to HIV infection and other STIs like gonorrhoea, chlamydia, syphilis, human papilloma virus (HPV) and herpes simplex virus (HSV) infections. There is evidence that increasing the availability of multiple contraceptive methods for women is associated with increased contraceptive uptake, lower pregnancy rates and fewer STIs. ${ }^{9}$ Furthermore, the contraceptive needs and preferences of women have been found to change over the course of their reproductive life, and it 
is imperative that women have a wide variety of options available to encourage them to use their contraceptive of choice. ${ }^{9}$ There are several methods of contraception that exist for women such as female sterilisation, long-acting hormonal contraceptives, short-acting hormonal contraceptives, copper intrauterine devices, barrier methods and natural method. ${ }^{10}$ However, the female condom, which is a barrier method of contraception, is the only female-initiated contraceptive method that offers dual protection against both pregnancy and STIs. In fact, there is evidence suggesting that it may be as effective as the male condom though this conclusion has not been demonstrated. ${ }^{1112}$

Introduced over two decades ago, the female condom offers the possibility of an alternative to male condoms. ${ }^{12}$ Several types of materials can be used to make female condoms, including polyurethanes, synthetic nitrile rubber latex, natural rubber latex and silicon. ${ }^{13}$ Generally, the structure of the female condom consists of a sheath that lines the vagina and may extend to cover the external genitalia. At the closed end of the sheath, a flexible ring of foam sponge is inserted into the vagina to hold the female condom in place. These internal retention features also help to facilitate insertion of the female condom into the vagina. At the other open end of the sheath, there is a ring or frame that stays outside the vulva at the entrance to the vagina. This ring or frame prevents the sheath bunching up inside the vagina and also facilitates removal of the condom. Some female condoms such as the Pheonurse are prelubricated and others like the Cupid are scented. ${ }^{13} 14$ The first-generation female condom, available since 1993, was made out of polyurethane. However, it has been progressively replaced by newer female condoms, designed to lower unit cost and/ or increase acceptability. Clinical studies evaluating the efficacy, safety and acceptability of these new designs are ongoing. ${ }^{15-18}$

In comparison with the male condom, the female condom is said to offer additional coverage to both partners and is not weakened by the use of oil-based lubricants. Furthermore, no serious local side effects or allergies have been reported. However, this non-systemic contraceptive method is not without limitations. It is known to be relatively more expensive, with mechanical problems which could include breakage, slippage, invagination and misdirection among others. ${ }^{19} 20$ These limitations are increasingly being addressed by the designing and manufacture of newer forms of the female condom, with emphasis on proper and frequent use of existing ones. ${ }^{1320}$

Research has been undertaken to determine the feasibility of reusing the female condom. ${ }^{21} \mathrm{~A}$ consultation convened by the WHO in January 2002 addressed certain considerations regarding the reuse of female condom. ${ }^{22}$ They concluded that although the use of a new female condom during each act of sexual intercourse should be recommended, the female condom can be reused in couples not at risk of pregnancy, STIs or HIV infection, but with careful attention to a disinfection, washing, drying and relubrication procedure. Research on the structural integrity of the female condom shows that it is maintained after five uses. ${ }^{23}$ However, additional research on the effectiveness of female condom reuse is still ongoing with newer designs of the female condom. ${ }^{13}$

There is evidence that condom use results in up to $80 \%$ reduction in the incidence of HIV. However, these estimates generally refer to the efficacy of the male condom. ${ }^{62425}$ With the advent of newer forms of the female condom, many randomised controlled studies have examined the effectiveness and acceptability of female condoms in preventing HIV and other STIs. ${ }^{15-18}$ Additionally, stereotypes and strong opinions that tend to hamper the acceptance of female condoms exist. These in turn may hinder their correct and consistent use, an aspect that determines the effectiveness of this barrier method in preventing HIV. In this review, we seek to examine the evidence from both randomised and non-randomised trials, on the effect of female condom use on the incidence of HIV and other STIs among women. We also plan to explore the side effects and acceptability of female condoms.

\section{METHODS AND ANALYSIS}

\section{Patient and public involvement}

Patients were not involved in the design of this study. However, the development of the research question and outcome measures were informed by patient's priorities, experience and preferences as reported in the literature supporting this review. The findings of this review will provide patients and policy-makers with the evidence on the efficacy and safety of existing and newer types of female condoms.

\section{Criteria for considering studies for this review}

We will include randomised and non-randomised trials that enrolled HIV negative and/or HIV positive women, engaged in heterosexual activity in any setting, with no clinical or laboratory-confirmed signs of STIs.

In addition, eligible trials would be those that compared the female condom to no treatment or other barrier methods for HIV prevention, for example, male condom, microbicides, diaphragm, vaginal sponges and cervical caps.

Finally, eligible studies need to report at least one of our primary or secondary outcomes of interest. Our primary outcomes for this review include acquisition of HIV (determined by a serological test) or STIs (including, but not limited to chlamydia, gonorrhoea, syphilis, HSV, trichomoniasis, candidiasis, lymphogranuloma venereum, HPV and bacterial vaginosis). Eligible studies need to determine STI status by microscopy and/or culture of urogenital specimens and vesicle fluid (when possible) for the causal agents. We will also consider nucleic acid amplification tests, if reported. Cytological pap testing used to determine human papillomavirus infection, and microscopy of Gram stained genital smear used to 
detect bacterial vaginosis will be acceptable methods of determining status. We will also include studies in which STIs were diagnosed clinically, and subgroup analyse by method of diagnosis (clinical vs laboratory).

Our secondary outcomes will include acceptability and adverse events of female condom use. Measures of acceptability may include scales to grade acceptability and where possible, these will be standardised to allow for quantitative comparison across trials. If this is not possible, then we will provide a narrative synthesis. Adverse events may include difficulties in insertion and removal of the condom leading to inconsistent use, breakage and slippage of condom, decreased pleasure and penetration difficulties during intercourse, genital ulcerations during intercourse and any other adverse events reported in the trials.

\section{Search methods for identification of studies}

We will use keywords to build a comprehensive search strategy that will be used to search the Cochrane Central Register of Controlled Trials, PubMed, EMBASE and Scopus for publications indexed from 1980 to July 2018. We have chosen to limit our search to this timeline as it corresponds to the identification of the first case of HIV. We have provided the proposed search strategy for one database, PubMed, in table 1 . We will also search the WHO International Clinical Trials Registry Platform for ongoing studies and the reference lists of included studies and related reviews for other relevant studies. We will include trial reports available in English or French.

\section{Study selection}

We will develop the search strategy and conduct the electronic searches with the help of an information specialist. The search output from the various databases will be combined and deduplicated using a reference management software (EndNote). Two authors will independently screen the titles and abstracts obtained from the electronic searches to create a pool of potentially eligible studies. Disagreement between the two authors will be resolved by discussion and consensus, and a third author will arbitrate if discussions fail. We will obtain the full articles of the potentially eligible studies which two authors will independently scrutinise for relevance using a standardised eligibility form with predefined inclusion criteria. The criteria for relevance will be based on the study design, interventions, participants and outcomes. If some of the information needed to classify the study is missing, we will attempt to contact the study authors for clarification. In the event where the authors do not have the missing information, or fail to respond, the study will be classified as 'awaiting assessment'. Disagreements between the two authors will be resolved by discussion and consensus. Should the disagreement persist, a third author will arbitrate. Following the eligibility assessment, each study will be classified as included, excluded, ongoing or awaiting classification. A study that meets the design, intervention and participant criteria for which

\begin{tabular}{|c|c|}
\hline Search & Query \\
\hline$\# 1$ & $\begin{array}{l}\text { Search (HIV Infections[MeSH] OR HIV[MeSH] } \\
\text { OR hiv[tiab] OR hiv-1*[tiab] OR hiv-2*[tiab] OR } \\
\text { hiv1[tiab] OR hiv2[tiab] OR hiv infect*[tiab] OR } \\
\text { HIV[tiab] OR human immunedeficiency virus[tiab] } \\
\text { OR human immuno-deficiency virus[tiab] OR } \\
\text { human immune-deficiency virus(tiab) OR ((human } \\
\text { immun [tiab]) AND (deficiency virus[tiab])) OR } \\
\text { AIDS[tiab] OR acquired immunedeficiency } \\
\text { syndrome[tiab] OR acquired immuno-deficiency } \\
\text { syndrome[tiab] OR acquired immune-deficiency } \\
\text { syndrome[tiab] OR ((acquired immun*[tiab]) AND } \\
\text { (deficiency syndrome[tiab])) }\end{array}$ \\
\hline \#2 & 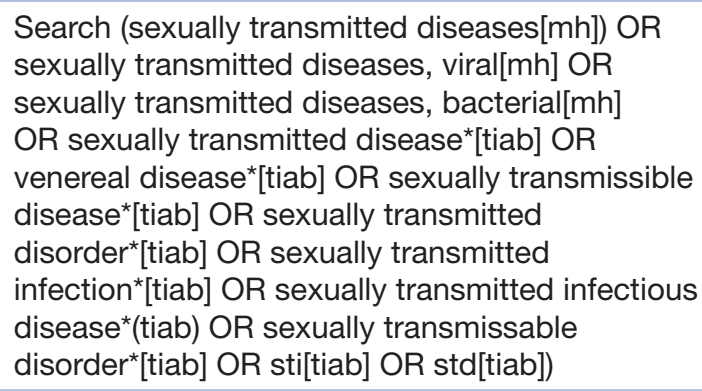 \\
\hline \#3 & Search (\#1 OR \#2) \\
\hline \#4 & $\begin{array}{l}\text { Search (randomized controlled trial [pt] OR } \\
\text { controlled clinical trial [pt] OR randomized [tiab] } \\
\text { OR placebo [tiab] OR drug therapy [sh] OR } \\
\text { randomly [tiab] OR trial [tiab] OR groups [tiab]) } \\
\text { NOT (animals [mh] NOT humans [mh]) }\end{array}$ \\
\hline \#5 & $\begin{array}{l}\text { Search (condoms, female[mh] OR female } \\
\text { condom[tiab] OR female condoms[tiab] OR } \\
\text { (female[tiab] AND condom*[tiab])) }\end{array}$ \\
\hline \#6 & Search (\#3 AND \#4 AND \#5) \\
\hline
\end{tabular}

relevant outcomes are not yet available will be classified as ongoing (if the study is not yet completed) or awaiting classification (if already completed). We will prepare a table of the excluded studies, with reasons for exclusion. All four authors will take part in study selection.

\section{Data extraction}

Two authors will independently extract data using a standard data extraction form. Extracted information will include study details such as location and setting, study design, population size and attrition rate; intervention details such as time period for the intervention and length of follow-up; comparator details including the type of comparator, time period for the comparator and length of follow-up; and outcome details such as HIV and STI incidence (with types of laboratory tests used to confirm HIV and STI diagnosis), degree of compliance with female condom use, acceptability and adverse effects. Disagreement between the two authors will be resolved by discussion and consensus. Should the disagreement persist, a third author will arbitrate. Where information in the study report(s) is unclear or missing, we will contact the authors and request for the missing information. If the 
authors fail to provide the missing information, the study will be included in the review; however, the findings that are unavailable will not be synthesised with findings from other included studies addressing the relevant outcome.

\section{Assessing risk of bias}

Two authors will independently assess the risk of bias in each included trial using the Cochrane risk of bias tool. ${ }^{26}$ This will include information on the adequacy of the generation of the allocation sequence and allocation concealment (for assessment of the risk of selection bias), blinding care providers (for performance bias), blinding of outcome assessors (for detection bias), completeness of outcome data (for attrition bias), completeness of outcome reporting (for reporting bias). Given the nature of the interventions considered in this review, the study participants cannot be blinded. The risk of performance bias will therefore be assessed based on whether the care providers were aware of the intervention or not.

\section{Data synthesis}

We will use the Cochrane Review Manager for data analyses. We will express study results as risk ratios (for dichotomous variables such as HIV incidence) or mean differences (for continuous outcomes such as acceptability); with their $95 \%$ CIs. We will combine study results in a meta-analysis if included trials found are similar in terms of design, participants, interventions and outcomes.

We will assess heterogeneity between trial results by visually inspecting the forest plots to assess whether the CIs overlap, followed by a more formal test, that is, the $\chi^{2}$ test of homogeneity (with significance defined as an alpha level of $10 \%$ ). We will also use the $\mathrm{I}^{2}$ test to quantify the degree of heterogeneity.

In the absence of significant statistical heterogeneity, we will pool the study results using the Mantel-Haenszel fixed-effect method. If we detect significant heterogeneity and consider it clinically meaningful to combine the trials, we will use the random-effects meta-analysis. We will explore the cause of observed heterogeneity using subgroup analyses, with subgroups defined by study design (randomised vs non-randomised trials), HIV status (for outcomes other than HIV acquisition), method of STI diagnosis (clinical vs laboratory), type of comparison intervention, trial duration, degree of compliance with female condom use and sample size. When a significant statistical association is found, we shall calculate the absolute risk reduction (or increase) with the number needed to treat or number needed to harm, as appropriate. Data obtained from studies that are not similar enough to be meta-analysed will be combined using narrative synthesis. We will use a funnel plot to asses for publication bias if we have more than 10 included studies in a meta-analysis. Finally, we will assess the strength or certainty of the evidence using the Grading of Recommendations Assessment, Development and Evaluation approach which rates the certainty of evidence for each outcome by taking into consideration the risk of bias, directness of evidence, heterogeneity, precision and risk of publication bias. ${ }^{27}$

\section{Timeline for the systematic review}

We registered the planned systematic review with the International Prospective Register of Systematic Reviews (PROSPERO) in March 2018. ${ }^{28}$ We plan to finalise the search strategy in August 2018, conduct the searches and select eligible studies between August and October 2018; and collect data, conduct statistical analyses, and prepare and submit the manuscript for consideration by a peer-reviewed journal between November 2018 and April 2019.

\section{Ethics and dissemination}

We will use data that are readily available in the public domain, hence no formal ethical approval is required for this review. The findings of this review will be presented at relevant conferences and published in a peer-reviewed journal. This protocol has been written following the Preferred Reporting Items for Systematic Reviews and Meta-Analyses Protocols guidelines, ${ }^{29}$ and the findings of this review and any amendments will be reported according to the Preferred Reporting Items for Systematic Reviews and Meta-Analyses statement. ${ }^{30}$

\section{Author affiliations}

${ }^{1}$ Department of Internal Medicine, Ottumwa Regional Health Center, Ottumwa, lowa, USA

${ }^{2}$ Cochrane South Africa, South African Medical Research Council, Cape Town, Western Cape, South Africa

${ }^{3}$ School of Global Health and Bioethics, Euclid University, Banjul, Gambia ${ }^{4}$ Division of Epidemiology and Biostatistics, Department of Global Health, Stellenbosch University, Cape Town, South Africa

${ }^{5}$ Division of Epidemiology and Biostatistics, School of Public Health and Family Medicine, University of Cape Town, Cape Town, South Africa

Contributors RKB conceived the study and wrote the first draft of the protocol ABW, EJK and CSW provided content and methodological expertise. All authors read, amended and approved the final version of the protocol before submission. CSW is the guarantor for this review.

Funding This review will be supported by the South African Medical Research Council and the National Research Foundation of South Africa (Grant Number: 108571).

Disclaimer The sponsors played no role in the design of the protocol, writing of the report and in the decision to submit the protocol for publication.

Competing interests None declared.

Patient consent Not required.

Provenance and peer review Not commissioned; externally peer reviewed.

Open access This is an open access article distributed in accordance with the Creative Commons Attribution Non Commercial (CC BY-NC 4.0) license, which permits others to distribute, remix, adapt, build upon this work non-commercially, and license their derivative works on different terms, provided the original work is properly cited, appropriate credit is given, any changes made indicated, and the use is non-commercial. See: http://creativecommons.org/licenses/by-nc/4.0/.

\section{REFERENCES}

1. Hay SI, Abajobir AA, Abate $\mathrm{KH}$, et al. Global, regional, and national disability-adjusted life-years (DALYs) for 333 diseases and injuries and healthy life expectancy (HALE) for 195 countries and territories, 1990-2016: a systematic analysis for the Global Burden of Disease Study 2016. Lancet 2017;390:1260-344. 
2. Wang $\mathrm{H}$, Wolock TM, Carter $\mathrm{A}$, et al. Estimates of global, regional, and national incidence, prevalence, and mortality of HIV, 19802015: the Global Burden of Disease Study 2015. Lancet HIV 2016;3:e361-e387.

3. Ward H, Rönn M. Contribution of sexually transmitted infections to the sexual transmission of HIV. Curr Opin HIV AIDS 2010;5:305-10.

4. Arora P, Nagelkerke NJ, Jha P. A systematic review and metaanalysis of risk factors for sexual transmission of HIV in India. PLOS One 2012;7:e44094.

5. Chun HM, Carpenter RJ, Macalino GE, et al. The Role of Sexually Transmitted Infections in HIV-1 Progression: A Comprehensive Review of the Literature. J Sex Transm Dis 2013;2013:1-15.

6. Weller SC, Davis-Beaty K. Condom effectiveness in reducing heterosexual HIV transmission. Chichester, UK: John Wiley \& Sons, Ltd, 2002. doi.

7. Madiba S, Ngwenya N. Cultural practices, gender inequality and inconsistent condom use increase vulnerability to HIV infection: narratives from married and cohabiting women in rural communities in Mpumalanga province, South Africa. Glob Health Action 2017;10:1341597.

8. Dube N, Nkomo TS, Khosa P. Condom Usage Negotiation Among Customarily Married Women in Katlehong, Johannesburg. Sage Open 2017;7:215824401668734.

9. Gray A, Smit J, Manzini N, et al. Systematic review of contraceptive medicines 'Does choice make a difference?' Reprod Heal HIV Res Unit. 2006:1-58.

10. Mansour D, Inki P, Gemzell-Danielsson K. Efficacy of contraceptive methods: A review of the literature. Eur J Contracept Reprod Health Care 2010;15:4-16.

11. Gallo MF, Kilbourne-Brook M, Coffey PS. A review of the effectiveness and acceptability of the female condom for dual protection. Sex Health 2012;9:18.

12. Health R. Female Condom Technical Review Committee, 2006:18-21.

13. Beksinska M, Smit J, Joanis C, et al. New female condoms in the pipeline. Reprod Health Matters 2012;20:188-96.

14. World Health Organization UNPF and F. Female Condom : Generic Specification, Prequalification and Guidelines for Procurement, 2012.

15. Joanis C, Beksinska M, Hart C, et al. Three new female condoms: which do South-African women prefer? Contraception 2011;83:248-54.

16. Beksinska M, Greener R, Kleinschmidt I, et al. A randomized noninferiority crossover controlled trial of the functional performance and safety of new female condoms: an evaluation of the Velvet, Cupid2, and FC2. Contraception 2015;92:261-7.

17. Beksinska ME, Piaggio G, Smit JA, et al. Performance and safety of the second-generation female condom (FC2) versus the Woman's, the VA worn-of-women, and the Cupid female condoms: a randomised controlled non-inferiority crossover trial. Lancet Glob Health 2013;1:e146-e152.

18. Ting RS, Wong EL, Tnay JK. A pilot study on the functional performance and acceptability of an innovative female condom (Wondaleaf ${ }^{\circledR}$ ) in Malaysia. Open Access $J$ Contracept 2018:9:11-20.

19. Nelson R. Female-initiated prevention strategies key to tackling HIV. Lancet Infect Dis 2007;7:637.

20. Beksinska M, Smit J, Joanis C, et al. Practice makes perfect: reduction in female condom failures and user problems with short-term experience in a randomized trial. Contraception 2012;86:127-31

21. Smith JB, Nkhama G, Trottier DA. Female condom reuse in Lusaka, Zambia: evidence from 12 cases. J Urban Health 2001;78:638-46.

22. WHO Information Update: Considerations regarding Reuse of the Female Condom, 2002.

23. Potter B, Gerofi J, Pope M, et al. Structural integrity of the polyurethane female condom after multiple cycles of disinfection, washing, drying and relubrication. Contraception 2003;67:65-72.

24. Liu H, Su Y, Zhu L, et al. Effectiveness of ART and condom use for prevention of sexual HIV transmission in serodiscordant couples: a systematic review and meta-analysis. PLoS One 2014;9:e111175.

25. Giannou FK, Tsiara CG, Nikolopoulos GK, et al. Condom effectiveness in reducing heterosexual HIV transmission: a systematic review and meta-analysis of studies on HIV serodiscordant couples. Expert Rev Pharmacoecon Outcomes Res 2016;16:489-99.

26. Higgins JP, Altman DG, Gøtzsche PC, et al. The Cochrane Collaboration's tool for assessing risk of bias in randomised trials. BMJ 2011;343:d5928.

27. Balshem $\mathrm{H}$, Helfand $M$, Schünemann HJ, et al. GRADE guidelines: 3. Rating the quality of evidence. J Clin Epidemiol 2011;64:401-6.

28. Bame R, Wiyeh A, Kongnyuy $\mathrm{E}$, et al. The effectiveness of the female condom in preventing HIV and sexually transmitted infections: a systematic review protocol. PROSPERO 2018 http://www.crd. york.ac.uk/PROSPERO/display_record.php?ID=CRD42018090710 (accessed 25 May 2018).

29. Shamseer L, Moher D, Clarke M, et al. PRISMA-P Group. Preferred reporting items for systematic review and meta-analysis protocols (PRISMA-P) 2015: elaboration and explanation. BMJ 2015;350:g7647.

30. Liberati A, Altman DG, Tetzlaff J, et al. The PRISMA statement for reporting systematic reviews and meta-analyses of studies that evaluate health care interventions: explanation and elaboration. PLoS Med 2009;6:e1000100. 